\title{
PET imaging of the mouse brain reveals a dynamic regulation of SERT density in a chronic stress model
}

\author{
Sonali N. Reisinger', Thomas Wanek², Oliver Langer, 2,3,4 and Daniela D. Pollak ${ }^{1}$
}

\begin{abstract}
The serotonin transporter (SERT, Slc6a4) plays an important role in the regulation of serotonergic neurotransmission and its aberrant expression has been linked to several psychiatric conditions. While SERT density has been proven to be amenable to in vivo quantitative evaluation by positron emission tomography (PET) in humans, this approach is in its infancy for rodents. Here we set out to evaluate the feasibility of using small-animal PET employing $\left[{ }^{11} \mathrm{C}\right] \mathrm{DASB}\left(\left[{ }^{11} \mathrm{C}\right]-\right.$ 3-amino-4-(2-dimethylaminomethyl-phenylsulfanyl)-benzonitrile) as a radiotracer to measure SERT density in

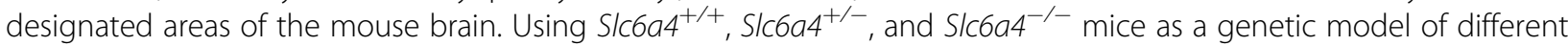
SERT expression levels, we showed the feasibility of SERT imaging in the mouse brain with $\left[{ }^{11} \mathrm{C}\right] \mathrm{DASB}-\mathrm{PET}$. The PET analysis was complemented by an evaluation of SERT protein expression using western blot, which revealed a highly significant correlation between in vivo and ex vivo measurements. $\left[{ }^{11} \mathrm{C}\right]$ DASB-PET was then applied to the examination of dynamic changes of SERT levels in different brain areas in the chronic corticosterone mouse model of chronic stress. The observed significant reduction in SERT density in corticosterone-treated mice was independently validated by and correlated with western blot analysis. This is the first demonstration of a quantitative in vivo evaluation of SERT density in subregions of the mouse brain using $\left[{ }^{11} \mathrm{C}\right] \mathrm{DASB}-\mathrm{PET}$. The evidenced decrease in SERT density in response to chronic corticosterone treatment adds a new dimension to the complex involvement of SERT in the pathophysiology of stressinduced mental illnesses.
\end{abstract}

\section{Introduction}

The serotonergic neurotransmitter system comprises a diffuse neuronal network that is involved in the control of several fundamental brain functions, including the regulation of mood $^{1-3}$, sleep/wake rhythms ${ }^{4,5}$, aggression ${ }^{6}$, appetite $^{7,8}$, learning and memory, and reward ${ }^{9-11}$. Correspondingly, alterations in serotonergic neurotransmission are implicated in a wide range of mental illnesses, from mood and anxiety to substance abuse disorders ${ }^{12,13}$

Correspondence: Daniela D. Pollak (daniela.pollak@meduniwien.ac.at) 1 Department of Neurophysiology and Neuropharmacology, Center for Physiology and Pharmacology, Medical University of Vienna,

Schwarzspanierstraße 17, 1090 Vienna, Austria

${ }^{2}$ Biomedical Systems, AIT Austrian Institute of Technology GmbH, 2444

Seibersdorf, Austria

Full list of author information is available at the end of the article.

These authors contributed equally: SN Reisinger, T Wanek and many psychoactive medications impact on the serotonergic system.

The activity and kinetics of serotonergic neurotransmission are critically dependent upon reuptake of serotonin $(5-\mathrm{HT})$ from the synaptic cleft into the presynaptic neuron by the serotonin transporter (SERT, SLC6A4). Over the last four decades, its important role as a major regulatory element of the 5-HT system has made SERT an attractive drug target for the development of psychoactive medications. Indeed, some of the most widely used pharmacological therapies for mood and anxiety disorders act upon SERT. Additionally, SERTgene polymorphisms and their relevance for sensory processing sensitivity ${ }^{14}$ have contributed to the notion of a complex involvement of SERT within the gene $x$

\section{(c) The Author(s) 2019}

(c) (i) Open Access This article is licensed under a Creative Commons Attribution 4.0 International License, which permits use, sharing, adaptation, distribution and reproduction cc) in any medium or format, as long as you give appropriate credit to the original author(s) and the source, provide a link to the Creative Commons license, and indicate if changes were made. The images or other third party material in this article are included in the article's Creative Commons license, unless indicated otherwise in a credit line to the material. If material is not included in the article's Creative Commons license and your intended use is not permitted by statutory regulation or exceeds the permitted use, you will need to obtain permission directly from the copyright holder. To view a copy of this license, visit http://creativecommons.org/licenses/by/4.0/. 
environment interactions shaping the susceptibility for the development of psychiatric disorders ${ }^{15-18}$.

However, despite more than 40 years of intensive research efforts the specific role of SERT in mental illness and the therapeutic response to psychotropic medications remains incompletely understood ${ }^{19,20}$. Limitations in the translatability from preclinical research to the human patient have posed a major obstacle both in basic research and drug development ${ }^{21,22}$. Methodologies that are amenable to human application as well as animal model experiments hold the potential to aid in bridging the gap from bench to bedside. Positron emission tomography (PET) is one of these methods, which can measure the kinetics of radiolabeled molecules (so-called radiotracers) in virtually all tissues of the body in a minimally invasive manner. With the availability of a constantly increasing toolbox of radiotracers that specifically bind to a wide array of different molecular target structures, PET holds great potential to monitor disease progression and treatment response. In the last two decades, the development of small-animal PET technology has allowed the examination of humans and rodents in comparable paradigms, allowing for a minimally invasive in vivo investigation of neural circuitries and molecular targets in longitudinal studies in patients and experimental animals. For quantification of SERT density in the brain, $\left[{ }^{11} \mathrm{C}\right]$ DASB (3amino-4-(2-dimethylaminomethylphenylsulfanyl)-benzonitrile $)^{23}$ has been most successfully used as a radiotracer in human studies and is currently considered the gold standard in the field ${ }^{24-26}$. However, the implementation of SERT-PET imaging in the translational neurosciences using animal models is a challenging task, as reflected in the difficulties to obtain accurate quantitative parameters of SERT binding in the mouse brain using a PET-based approach $^{27}$.

Here we set out to establish $\left[{ }^{11} \mathrm{C}\right] \mathrm{DASB}-\mathrm{PET}$ as a suitable tool for the in vivo monitoring of SERT density in the mouse brain with the aim to detect dynamic changes in SERT levels in response to environmental conditions favoring the development of psychiatric conditions in a validated animal model.

\section{Materials and methods \\ Chemicals}

Unless otherwise stated, all chemicals were purchased from Sigma-Aldrich (Schnelldorf, Germany) or Merck (Darmstadt, Germany).

\section{Radiotracer synthesis}

$\left[{ }^{11} \mathrm{C}\right]$ DASB was synthesized by $N$-methylation of $N$ desmethyl-DASB (ABX GmbH, Radeberg, Germany) with $\left[{ }^{11} \mathrm{C}\right]$ methyl iodide as described in the literature ${ }^{28,29}$. The entire synthesis procedure was fully automated in a Tracerlab FX C Pro synthesis module (GE Healthcare Uppsala, Sweden). $\left[{ }^{11} \mathrm{C}\right]$ DASB was formulated in physiological saline solution $(0.9 \%, \mathrm{w} / \mathrm{v})$ containing $0.1 \%(\mathrm{v} / \mathrm{v})$ polysorbate- 80 and a maximum of $5 \%(\mathrm{v} / \mathrm{v})$ ethanol for intravenous (i.v.) injection into animals. Radiochemical purity of $\left[{ }^{11} \mathrm{C}\right] \mathrm{DASB}$ was $>98 \%$. Molar activity at the time of radiotracer injection into different animal groups is reported in Table 1.

\section{Animals}

Heterozygous founder pairs for the generation of wildtype $\left(\operatorname{Slc} 6 a 4^{+/+}\right)$, heterozygous $\left(S l c 6 a 4^{+/-}\right)$, and homozygous $\left(\right.$ Slc6a4 $\left.{ }^{-1-}\right)$ SERT knockout mice on a C57BL/6J genetic background were provided by Harald $\mathrm{H}$. Sitte (Medical University of Vienna, Austria). Wild-type C57BL/6N mice used in the chronic corticosterone (CORT) paradigm were obtained from Charles River (Sulzfeld, Germany). For a summary of animal numbers used for PET scans in each part of this study see Table 1. All mice were single-housed for 1 week before the start of experiments, kept on a 12-h light-dark cycle, and had ad libitum access to food and water. At the time of the first PET scan, animals weighed $23.5 \pm 2.7 \mathrm{~g}$.

A habituation period of at least 1 week was scheduled after the transfer to the preclinical imaging facility (Austrian Institute of Technology; Seibersdorf, Austria) and before the animals were subjected to PET scans. The study was approved by the national authorities (Amt der Niederösterreichischen Landesregierung) and study procedures were in accordance with the European Communities Council Directive of September 22, 2010 (2010/63/EU).

\section{Experimental design}

An overview of the experimental design of this study is depicted in Fig. 1. In the first set of experiments (part A),

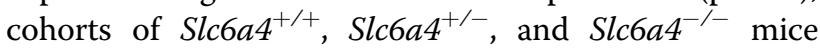
underwent $\left[{ }^{11} \mathrm{C}\right] \mathrm{DASB}-\mathrm{PET}$ scans, followed by brain extraction and ex vivo determination of hippocampal SERT expression by western blot. In a second set of experiments (part B), wild-type mice underwent a baseline $\left[{ }^{11} \mathrm{C}\right] \mathrm{DASB}-\mathrm{PET}$ scan prior to being randomly assigned to either treatment with corticosterone (CORT group) or water (CTRL group) for $57 \pm 2$ days after which animals underwent a second $\left[{ }^{11} \mathrm{C}\right] \mathrm{DASB}-\mathrm{PET}$ scan. Subsequently, animals were sacrificed and post-treatment SERT expression was biochemically examined by western blot. A parallel cohort of mice was subjected to chronic CORT treatment and tested for depression-related anxiety using the novelty-suppressed feeding test (NSF) in order to validate the potential of the chronic CORT paradigm to induce depression-like behavior. 
Table 1 Overview of examined animals, groups, and numbers investigated with [ $\left.{ }^{11} \mathrm{C}\right] \mathrm{DASB}-\mathrm{PET}$

\begin{tabular}{|c|c|c|c|c|c|}
\hline Genotype & Number/sex & Body weight (g) & Injected activity (MBq) & Molar activity ${ }^{\mathrm{a}}(\mathrm{GBq} / \mu \mathrm{mol})$ & Injected dose $(\mathrm{nmol} / \mathrm{kg})$ \\
\hline \multicolumn{6}{|l|}{ Part A } \\
\hline \multirow[t]{2}{*}{ Wild type $\left(S / c 6 a 4^{+/+}\right)$} & $8 /$ male & $24.9 \pm 2.3$ & $22.5 \pm 18.8$ & $210.6 \pm 101.3$ & $3.9 \pm 2.6$ \\
\hline & 5/female & $22.1 \pm 2.3$ & $40.4 \pm 3.7$ & $240.2 \pm 173.5$ & $12.6 \pm 9.6$ \\
\hline \multirow[t]{2}{*}{$S / c 6 a 4^{+/-}$} & $5 / \mathrm{male}$ & $25.8 \pm 2.3$ & $34.5 \pm 5.1$ & $331.4 \pm 97.5$ & $4.4 \pm 1.5$ \\
\hline & 5/female & $19.5 \pm 1.5$ & $37.6 \pm 5.8$ & $271.7 \pm 96.4$ & $7.8 \pm 2.5$ \\
\hline \multirow[t]{2}{*}{$S / c 6 a 4^{-/-}$} & $5 / \mathrm{male}$ & $25.7 \pm 4.1$ & $33.6 \pm 4.7$ & $261.0 \pm 183.8$ & $7.3 \pm 4.1$ \\
\hline & 4/female & $19.0 \pm 1.8$ & $35.5 \pm 4.1$ & $333.2 \pm 331.7$ & $10.3 \pm 6.7$ \\
\hline $\begin{array}{l}\text { Wild type }\left(S / c 6 a 4^{+/+}\right) \\
\text {low molar activity }\end{array}$ & 4/male & $27.2 \pm 1.2$ & $29.0 \pm 3.2$ & $48.9 \pm 10.4$ & $152.8 \pm 12.1$ \\
\hline \multicolumn{6}{|l|}{ Part B } \\
\hline \multicolumn{6}{|l|}{ Wild type } \\
\hline Baseline PET & 28/male & $23.8 \pm 1.1$ & $6.2 \pm 1.6$ & $73.2 \pm 13.5$ & $3.8 \pm 1.7$ \\
\hline \multicolumn{6}{|l|}{ Post-treatment PET } \\
\hline CORT & 20/male & $28.9 \pm 1.6$ & $5.64 \pm 0.91$ & $75.5 \pm 26.3$ & $2.94 \pm 1.20$ \\
\hline CTRL & $8 /$ male & $29.7 \pm 2.3$ & $5.24 \pm 0.58$ & $57.1 \pm 14.6$ & $3.34 \pm 1.25$ \\
\hline
\end{tabular}

Slc6a4 gene encoding for the serotonin transporter, CORT corticosterone treatment, CTRL control/vehicle treatment

${ }^{a}$ At the time of radiotracer injection into the animals

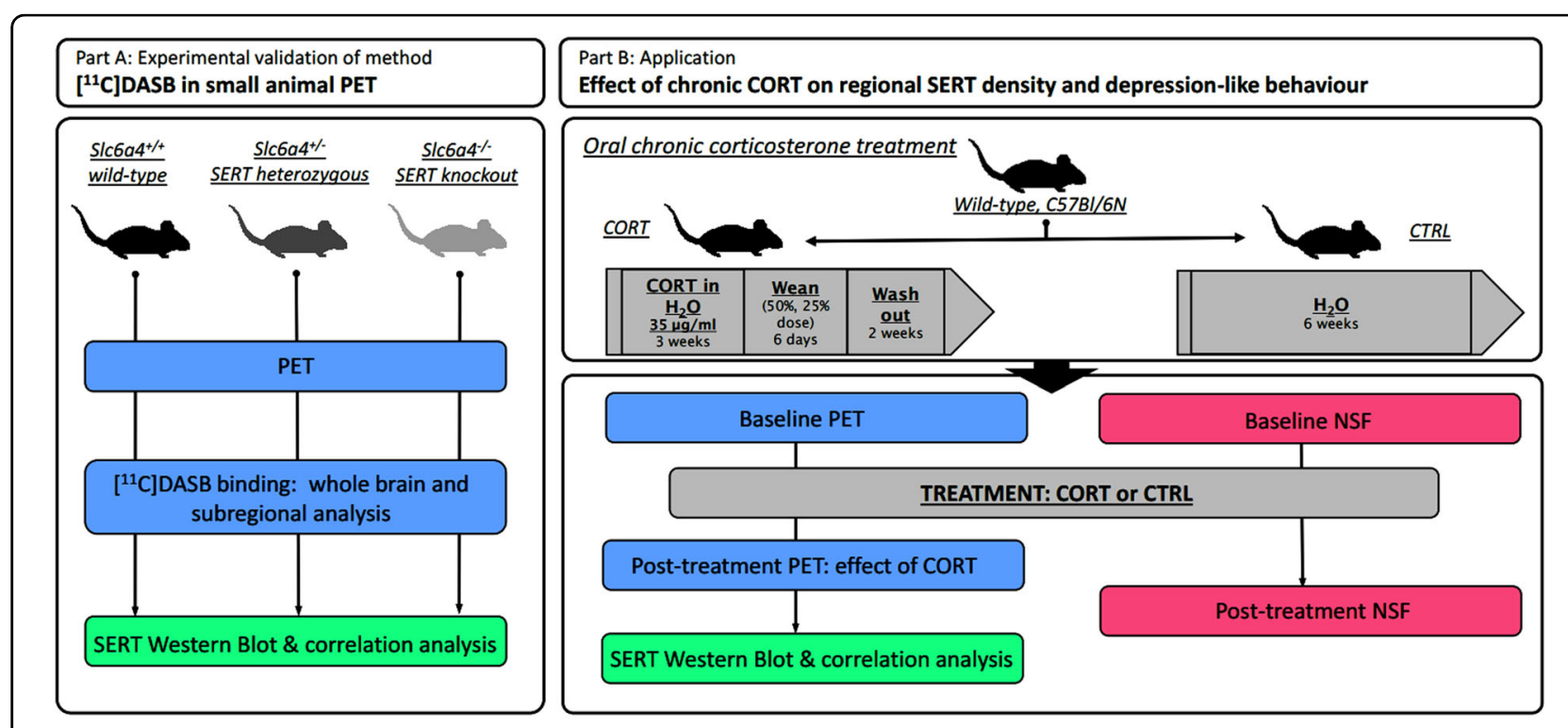

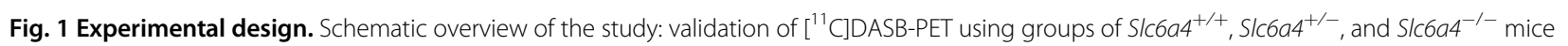
(part A) and investigation of the effects of a chronic CORT paradigm on SERT density as measured by $\left[{ }^{11} \mathrm{C}\right] \mathrm{DASB}-\mathrm{PET}$ and western blot, as well as depression-like behavior (part B). $\left[{ }^{11} \mathrm{C}\right] \mathrm{DASB}\left[\left[^{11} \mathrm{C}\right]-3\right.$-amino-4-(2-dimethylaminomethyl-phenylsulfanyl)-benzonitrile, PET positron emission tomography, SIc6a4/SERT serotonin transporter, NSF novelty-suppressed feeding test, CORT corticosterone treatment, CTRL control/vehicle treatment

\section{Chronic corticosterone treatment}

Mice were exposed to corticosterone-21hemisuccinate $\quad(4-[2-[(8 S, 9 S, 10 R, 11 S, 13 S, 14 S, 17 S)-11-$ hydroxy-10,13-dimethyl-3-oxo-1,2,6,7,8,9,11,12,14,15, 16,17-dodecahydrocyclopenta[a]phen-anthren-17-yl]-2oxoethoxy]-4-oxobutanoic acid; $35 \mu \mathrm{g} / \mathrm{mL}$ free base; Steraloids Inc., Newport, RI, USA) via the drinking water according to a published protocol ${ }^{30}$ with minor adaptations. Briefly, the CORT treatment lasted 3 weeks, followed by a weaning phase during which mice received a solution at $50 \%$ and then $25 \%$ of the full dose for 3 days respectively, with a subsequent washout period of 2 weeks to allow for the investigation of long-lasting effects of chronic $\mathrm{CORT}^{30}$. Control animals (CTRL) received regular drinking water for the entire duration of the experimental period. 


\section{Novelty-suppressed feeding test}

Mice ( $n=7-8$ /group) were weighed and food-restricted $24 \mathrm{~h}$ prior to behavioral testing. On the day of testing, they were habituated to the experimental room for $45 \mathrm{~min}$. A single food pellet was placed into the center of a brightly lit $(800 \mathrm{lux})$ arena $(30 \times 50 \mathrm{~cm})$ filled with wood-chip bedding material. The latency to feed on the pellet (s) was recorded for each mouse and used as a parameter to assess depression-related anxiety ${ }^{31,32}$. Mice that lost more than $20 \%$ of their body weight during the food restriction period were excluded from the analysis.

\section{PET imaging}

Imaging experiments were performed under isoflurane anesthesia (1.5-3.0\% in oxygen). Animals were warmed throughout the experiment and body temperature and respiratory rate were constantly monitored. Mice were placed in an imaging chamber and the lateral tail vein was cannulated for i.v. administrations. A microPET Focus220 scanner (Siemens Medical Solutions, Knoxville, TN, USA) was used for PET imaging. Dynamic emission scans $(90 \mathrm{~min})$ were started with the i.v. injection of $\left[{ }^{11} \mathrm{C}\right]$ DASB (see Table 1 for injected doses in individual animal groups). To assess the dose-dependency of SERT binding of $\left[{ }^{11} \mathrm{C}\right] \mathrm{DASB}$, unlabeled DASB (ABX GmbH, Radeberg, Germany) was co-injected with the radiotracer in a group of wild-type animals $(n=4$, Table 1$)$. List-mode data were acquired with a timing window of $6 \mathrm{~ns}$ and an energy window of $250-750 \mathrm{keV}$. For part A, at the end of the PET scan, a final blood sample $(20-40 \mu \mathrm{L})$ was collected from the retro-orbital venous plexus and animals were sacrificed by cervical dislocation under deep anesthesia. Brains were extracted and blood was centrifuged $\left(13,000 \times g, 4^{\circ}\right.$ $\mathrm{C}, 4 \mathrm{~min}$ ) to obtain plasma. Aliquots of blood and plasma were transferred into pre-weighed tubes and measured for radioactivity in a gamma counter (Wizard 1470, PerkinElmer, Wellesley, MA, USA). The measured radioactivity data were corrected for radioactive decay and expressed as standardized uptake values ((radioactivity per g/injected radioactivity) $\times$ body weight). For part $\mathrm{B}$, at the end of the baseline PET scan, a small blood sample $(10-15 \mu \mathrm{L})$ was collected and animals were placed back into their cages for repeated $\left[{ }^{11} \mathrm{C}\right] \mathrm{DASB}$ imaging following CORT or CTRL treatment. After the last PET scan, animals were sacrificed and processed as described above.

\section{Magnetic resonance imaging}

Anatomical magnetic resonance (MR) imaging was performed prior to PET imaging on a $1 \mathrm{~T}$ benchtop MR scanner (ICON, Bruker BioSpin GmbH, Ettlingen Germany) in a subset of animals $(n=8)$. For MR imaging a modified three-dimensional (3D) T1-weighted gradient echo sequence ( $\mathrm{T}_{1}$-FLASH) was used and the following imaging parameters: echo time $=4.7 \mathrm{~ms}$; repetition time $=27 \mathrm{~ms}$; flip angle $=25^{\circ}$; field of view $=7.6 \times 2.6 \times 2.4 \mathrm{~cm}$; matrix $=380 \times 130$; 32 slices; slice thickness $=750 \mu \mathrm{m}$; and total imaging time $=22 \mathrm{~min}$.

\section{PET data analysis}

The dynamic PET data were sorted into 25 frames, which incrementally increased in time length. PET images were reconstructed using Fourier re-binning of the 3D sinograms followed by a two-dimensional-filtered backprojection with a ramp filter giving a voxel size of $0.4 \times$ $0.4 \times 0.796 \mathrm{~mm}^{3}$. Using PMOD software (version 3.6, PMOD Technologies Ltd., Zurich, Switzerland), hippocampus, striatum, thalamus, cortex, and cerebellum were outlined on the PET images using the Mirrione Mouse Atlas and guided by representative MR images obtained in a few animals. The position of the regions of interest (ROIs) was manually adjusted if necessary without modification in ROI sizes. Following ROI definition, timeactivity curves (TACs) expressed in standardized uptake value units were derived. Regional PET data were modeled with the PKIN tool in PMOD using a simplified reference tissue model and whole cerebellum as a reference tissue to obtain the non-displaceable binding potential $\left(\mathrm{BP}_{\mathrm{nd}}\right)$ as an outcome parameter for SERTspecific binding of $\left[{ }^{11} \mathrm{C}\right] \mathrm{DASB}{ }^{27} . \mathrm{BP}_{\mathrm{nd}}$ is defined as the ratio between the available SERT density $\left(B_{\max }\right)$ and the apparent equilibrium dissociation constant $\left(K_{\mathrm{D}}\right)$ of the radiotracer. Under the assumption that $K_{\mathrm{D}}$ remains unchanged, $\mathrm{BP}_{\text {nd }}$ can be considered as a measure of SERT density. To assess the dose-dependency of SERT-specific binding of $\left[{ }^{11} \mathrm{C}\right] \mathrm{DASB}$ in wild-type $\left(S l c 6 a 4^{+/+}\right)$mice, a sigmoidal dose-response curve was fitted to a plot of $\mathrm{BP}_{\text {nd }}$ in the hippocampus, striatum, thalamus, and cortex to injected DASB dose $(\mathrm{nmol} / \mathrm{kg})$ using the following equation:

$$
B P_{n d}=B P_{n d, \max } \frac{\text { Dose }}{\left(E D_{50}+\text { Dose }\right)}
$$

where Dose is the injected DASB dose $(\mathrm{nmol} / \mathrm{kg}), \mathrm{ED}_{50}$ the half-maximum effect dose of DASB to displace SERTspecific binding of $\left[{ }^{11} \mathrm{C}\right]$ DASB $\left(\mathrm{nmol} / \mathrm{kg}\right.$ ), and $\mathrm{BP}_{\mathrm{nd}, \max }$ is the maximum $\mathrm{BP}_{\mathrm{nd}}$ value. A fixed Hill slope of -1.0 was assumed.

\section{Determination of reproducibility and reliability of $\left[{ }^{11} \mathrm{C}\right]$ DASB in CTRL group}

Test-retest analysis was performed as described in the literature ${ }^{33}$ by comparing $\mathrm{BP}_{\text {nd }}$ values in different brain regions at baseline with $\mathrm{BP}_{\text {nd }}$ values of the CTRL group measured approximately 57 days after baseline $(n=8)$. 
The test-retest variability (VAR) was calculated as follows:

$$
V A R=\frac{|\operatorname{scan} 2-\operatorname{scan} 1|}{0.5 \times(\operatorname{scan} 1+\operatorname{scan} 2)}
$$

where scan 2 represents the $\mathrm{BP}_{\text {nd }}$ values of the CTRL group and scan 1 represents the $\mathrm{BP}_{\text {nd }}$ values of the respective animals at baseline.

The test-retest reliability (RL) was calculated using the following formula:

$$
R L=\frac{S T D B^{2}}{\left(S T D B^{2}+S T D W^{2}\right)}
$$

where STDB represents the standard deviation between the subjects and STDW represents the standard deviations within the subject at both imaging time points.

\section{Western blot}

Immediately after the last PET scan, brains were rapidly extracted and the hippocampus, cerebellum, and cortex were dissected. Tissue samples were then snap-frozen in liquid nitrogen and stored at $-80{ }^{\circ} \mathrm{C}$ until used for western blot analysis.

Total protein was extracted from powderized brain tissue and $50 \mu \mathrm{g}$ of protein were loaded and subjected to SDS-polyacrylamide gel electrophoresis according to a previously described procedure ${ }^{34}$. Membranes were incubated overnight with the primary antibodies (SERT, target: sc-1458, dilution 1:500; secondary antibody: antigoat IgG-HRP, sc-2020, dilution 1:2000; both Santa Cruz Biotechnology, Inc, Dallas, TX, USA; $\beta$-actin, housekeeping protein: A0760-40, dilution 1:2000; US Biological, Swampscott, MA, USA; secondary antibody: anti-mouse IgG-HRP, \#7076, dilution 1:5000; Cell Signaling Technology, Danvers, MA, USA) used as the housekeeping protein. Chemiluminescent imaging was performed using a FluorChem HD2 imager (Alpha Innotec, Kasendorf, Germany) and densitometry values were determined using the software ImageJ (NIH, Bethesda, MD, USA). SERT protein densitometry values for each sample were normalized to the corresponding housekeeping protein value to obtain semiquantitative measures of SERT expression.

\section{Statistical analysis}

Differences between two groups were analyzed with a two-sided $t$-test (with Welch correction where applicable) and between multiple groups with two-way or one-way analysis of variance with post hoc Tukey's multiple comparison test using Prism 7 software (GraphPad Software, La Jolla, CA, USA). To assess correlations, the Pearson correlation coefficient $r$ was calculated. The level of statistical significance was set to a $P$ value of $<0.05$. All values are given as mean \pm standard deviation.

\section{Results}

In the present study we set out to examine dynamic changes in SERT density in the mouse brain using PET imaging with $\left[{ }^{11} \mathrm{C}\right] \mathrm{DASB}$ in a mouse model of chronic stress based upon long-term treatment with CORT. As $\left[{ }^{11} \mathrm{C}\right] \mathrm{DASB}$ had so far not been successfully used to monitor SERT density in the mouse brain in quantitative terms, we first conducted a comprehensive validation of $\left[{ }^{11} \mathrm{C}\right]$ DASB as a SERT-PET tracer in mice (see experimental design, Fig. 1, part A). To this end, we followed two different approaches. First, we examined SERT wildtype $\left(\operatorname{Slc} 6 a 4^{+/+}\right)$, heterozygous $\left(\operatorname{Slc} 6 a 4^{+/-}\right)$, and homozygous knockout $\left(S l c 6 a 4^{-/-}\right)$mice as genetic models of differential SERT expression. Second, as an additional validation step, we co-injected wild-type mice with unlabeled DASB to determine the $\mathrm{ED}_{50}$ for displacement of SERT-specific binding of $\left[{ }^{11} \mathrm{C}\right] \mathrm{DASB}$. We calculated the $\mathrm{BP}_{n d}$ as outcome parameter of SERT-specific binding of $\left[{ }^{11} \mathrm{C}\right]$ DASB using whole cerebellum as a SERT-free reference tissue ${ }^{27}$.

Considering the perspective of the application of $\left[{ }^{11} \mathrm{C}\right]$ DASB-PET for the evaluation of mouse models of neuropsychiatric disorders we strove to apply our analysis to the level of individual brain areas, by defining ROIs with relevance for SERT-related mental disorders. To this end, we used a mouse brain atlas in PMOD to outline the hippocampus, striatum, thalamus, and the cortex on PET images with the help of MR scans acquired in representative animals (Fig. 2a). TACs in these brain regions for the three mouse genotypes revealed clear visual differences, with highest values in Slc $6 a 4^{+/+}$, followed by Slc6a $4^{+/-}$and Slc6a4 ${ }^{-/-}$mice in the SERT-rich brain regions hippocampus, striatum, and thalamus (Supplementary Figure S1A-C). These differences were less pronounced in the cortex (Supplementary Figure S1D), which contains considerably lower SERT expression levels than the other three brain regions ${ }^{35}$. TACs of the three genotypes were almost superimposable in the cerebellum, designated as reference tissue, indicating very low SERT expression levels (Supplementary Figure S1E). $\mathrm{BP}_{\text {nd }}$ values in different brain regions for different mouse genotypes are summarized in Supplementary Table S1. No sex differences in $\mathrm{BP}_{\text {nd }}$ values were observed for any of the brain regions examined in the three genotypes (Supplementary Figure $\mathrm{S} 2 \mathrm{~A}-\mathrm{D})$. In all brain regions, $\mathrm{BP}_{\text {nd }}$ values of male mice

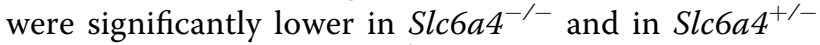
mice compared to Slc6a4 ${ }^{+/+}$mice. For female animals, significant differences between the three genotypes were found for hippocampus, striatum, and thalamus, but not for the cortex, which was most likely due to the weak 

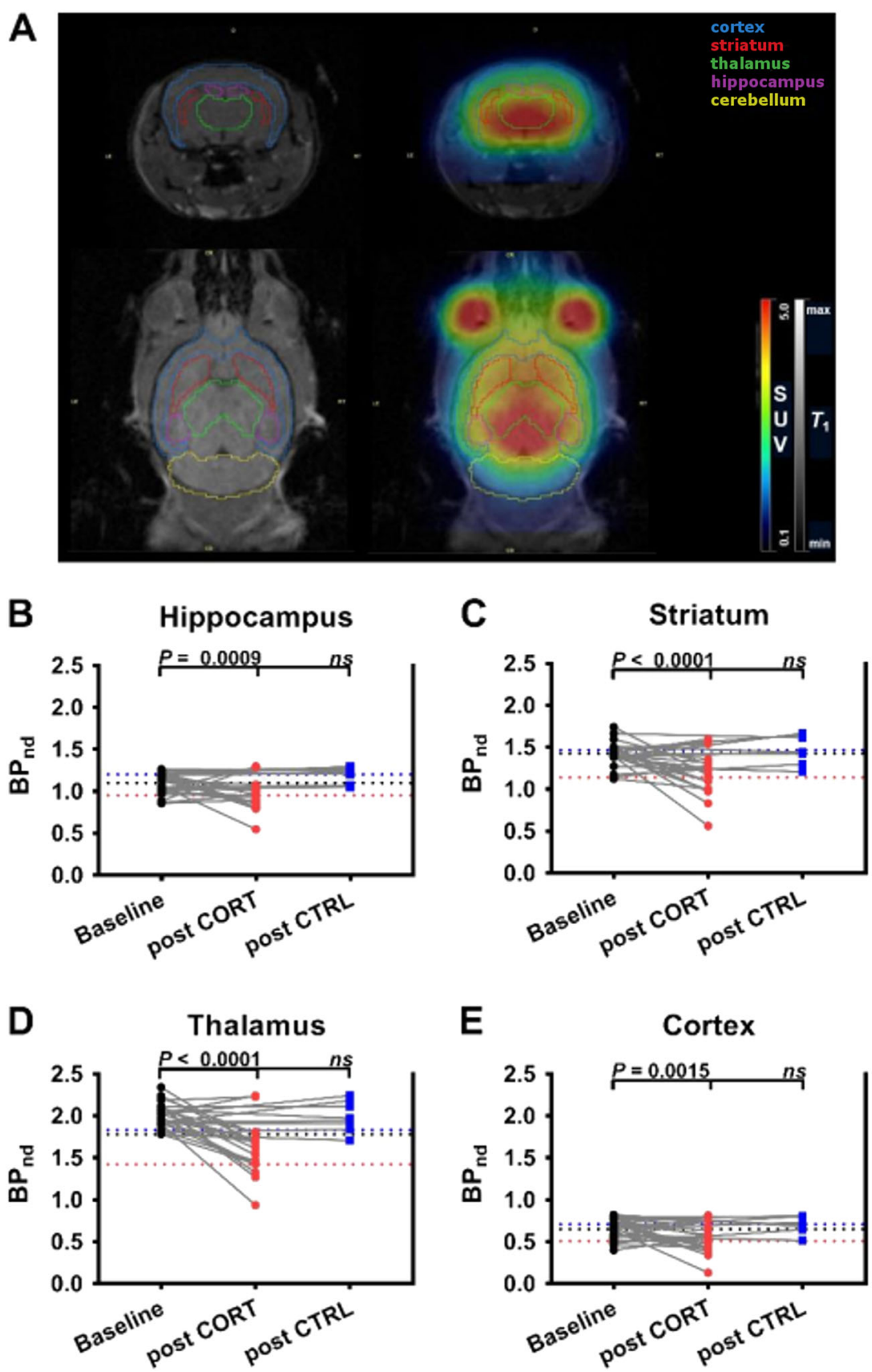

E

\section{Cortex}

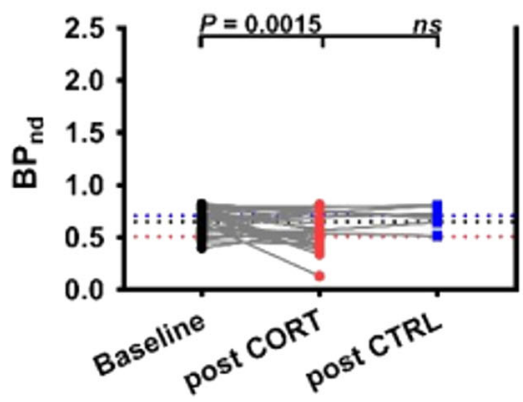

Fig. 2 BP $_{\text {nd }}$ values for CORT-treated and control groups. a Definition of brain ROls (hippocampus—-purple; striatum—red; thalamus—green; cortex — blue; cerebellum - yellow) for subregional analysis of SERT density on representative coronal and horizontal MR and MR co-registered PET summation images (0-90 min). $\mathrm{BP}_{\mathrm{nd}}$ values at baseline and after treatment with corticosterone (CORT group) or vehicle (CTRL group) in $\mathbf{b}$ hippocampus, $\mathbf{c}$ striatum, d thalamus, and e cortex of wild-type mice. Black dotted line indicates mean $\mathrm{BP}_{\mathrm{nd}}$ value of baseline scans, red dotted line indicates mean $\mathrm{BP}_{\text {nd }}$ value of second scan in CORT group, and blue dotted line indicates mean $\mathrm{BP}_{\text {nd }}$ value of second scan in CRTL group. One-way analysis of variance indicated a significant change in baseline $\mathrm{BP}_{\text {nd }}$ values following CORT treatment in hippocampus $\left(F_{2,52}=12.58, P<0.0001\right)$, striatum $\left(F_{2,52}=16.82, P<0.0001\right)$, thalamus $\left(F_{2,52}=20.51, P<0.0001\right)$, and cortex $\left(F_{2,52}=10.03, P=0.0002\right), n=8-27$. $P$ values indicate results of post hoc Tukey's multiple comparison analysis. Data are presented as paired individual values (gray lines) at baseline and after CORT or CTRL treatment. $\mathrm{BP}_{\text {nd }}$ non-displaceable binding potential, SUV standardized uptake value, ROI region of interest, CORT corticosterone treatment, CTRL control/vehicle treatment, ns nonsignificant, MR magnetic resonance, PET positron emission tomography 
SERT expression in this brain region making it difficult to assess variations in $\left[{ }^{11} \mathrm{C}\right] \mathrm{DASB}$ binding.

Blood concentration of radioactivity at the end of the PET scans was neither affected by genotype nor sex (Supplementary Figure S3).

This analysis was complemented by the examination of the dependency of $\mathrm{BP}_{\text {nd }}$ values on administered $\mathrm{DASB}$ dose (wild-type mice co-injected with unlabeled DASB) in all four brain regions (Supplementary Figure S4). The $\mathrm{ED}_{50}$ values of DASB to displace SERT-specific binding of $\left[{ }^{11} \mathrm{C}\right] \mathrm{DASB}$ in the different brain regions are given in Supplementary Table $\mathrm{S} 2$. For the thalamus, the $\mathrm{ED}_{50}$ value $(17.1 \pm 1.4 \mathrm{nmol} / \mathrm{kg})$ was in good agreement with the same value determined in a previous study in rats using cerebellar cortex as a reference tissue $(12.0 \pm 2.6 \mathrm{nmol} / \mathrm{kg})^{27}$.

We next sought to additionally validate the PET-based determination of SERT density by an independent method. We therefore resorted to ex vivo analysis by western blotting of the same mouse brains analyzed by PET. We specifically focused on the hippocampus, in light of its involvement in chronic stress and the pathophysiology of several SERT-related mental disorders ${ }^{36-38}$. A highly significant positive correlation of $\mathrm{BP}_{\text {nd }}$ values with relative SERT expression levels determined by western blot was revealed (Supplementary Figure S5), further corroborating the specificity of the PET-based evaluation of SERT density and validating its experimental usage for longitudinal studies in mice.

Having established and validated $\left[{ }^{11} \mathrm{C}\right] \mathrm{DASB}-\mathrm{PET}$ as a method for the quantitative assessment of SERT density in individual regions of the mouse brain, we moved on to its first application: the examination of dynamic changes of brain SERT levels in the chronic CORT mouse model of chronic stress. To this end, mice were either subjected to a long-term oral CORT treatment ("chronic stress") or received regular drinking water (CTRL). A parallel cohort of animals was used to verify the effect of CORT treatment on depression-related anxiety in the $\mathrm{NSF}^{39}$. We opted to not use the same animals in behavioral and imaging experiments in order to avoid effects of behavioral testing on SERT density. The animals of the imaging cohorts were scanned before (baseline) and after CORT and CTRL treatment, respectively (see experimental design, Fig. 1, part B). $\mathrm{BP}_{\text {nd }}$ values for the two PET scans in all four brain regions examined (Fig. 2a; hippocampus, striatum, thalamus, and cortex) revealed a significant decrease as compared to baseline in the CORT group but not in the CTRL group (Fig. 2b-e, Table 2). The mean percentage changes as compared to baseline scans in the CORT group were $-9.3 \pm 22.4 \%$ for the hippocampus, $-19.7 \pm 18.6 \%$ for the striatum, $-20.6 \pm$ $14.4 \%$ for the thalamus, and $-15.4 \pm 32.6 \%$ for the cortex. In 4 out of 20 animals of the CORT group $\mathrm{BP}_{\text {nd }}$ values were not decreased after CORT treatment but either
Table 2 Quantification of $\left[{ }^{11} \mathrm{C}\right] \mathrm{DASB}$ binding in brain regions of male wild-type mice at baseline $(n=28)$ and after CORT $(n=20)$ or CTRL $(n=8)$ treatment using a simplified reference tissue model with the whole cerebellum as reference tissue

\begin{tabular}{llll}
\hline $\begin{array}{l}\text { Region } \\
\text { Part B }\end{array}$ & $\begin{array}{lll}\mathrm{BP}_{\mathbf{n d}} \pm \mathrm{SD} \\
\text { Baseline }\end{array}$ & $\begin{array}{l}\mathrm{BP}_{\mathbf{n d}} \pm \mathrm{CD} \\
\mathbf{C O R T}\end{array}$ & $\begin{array}{l}\mathrm{BP}_{\mathbf{n d}} \pm \mathrm{SD} \\
\mathbf{C T R L}\end{array}$ \\
\hline Hippocampus & $1.10 \pm 0.12$ & $0.95 \pm 0.17^{\mathrm{a}}$ & $1.20 \pm 0.09$ \\
Striatum & $1.43 \pm 0.14$ & $1.14 \pm 0.24^{\mathrm{a}}$ & $1.47 \pm 0.17$ \\
Thalamus & $2.01 \pm 0.15$ & $1.61 \pm 0.30^{\mathrm{a}}$ & $1.99 \pm 0.18$ \\
Cortex & $0.65 \pm 0.12$ & $0.51 \pm 0.15^{\mathrm{a}}$ & $0.71 \pm 0.10$ \\
\hline
\end{tabular}

$B P_{n d}$ non-displaceable binding potential, CORT corticosterone treatment, CTRL control/vehicle treatment, $S D$ standard deviation

${ }^{a} P \leq 0.0002$, one-way analysis of variance with Tukey's multiple comparison test

remained similar or were slightly increased relative to baseline (Fig. 2b-e). CORT treatment did not affect blood radioactivity concentrations compared to baseline or CTRL (Supplementary Figure S6). We used the data acquired in the CTRL group to determine the test-retest VAR and RL of $\left[{ }^{11} \mathrm{C}\right]$ DASB-PET in different brain regions (see Supplementary Table S3).

Western blot analysis revealed a significant difference in SERT expression between groups post treatment (Fig. 3a, b). We additionally correlated hippocampal $\mathrm{BP}_{\text {nd }}$ values measured in the second PET scan with SERT levels determined by western blot and found a significant positive correlation between the outcome measures obtained by the two independent methodologies (Fig. 3c). In the behavioral cohort, both groups showed similar levels of depression-related anxiety at baseline, as assessed by the latency to start feeding in the NSF. However, as expected upon treatment ${ }^{39}$, CORT animals presented with a significantly augmented latency to retrieve the food pellet in the NSF in comparison to controls, in which the post-treatment latency was shorter than the pretreatment time to feed in the NSF possibly due to a degree of habituation to the testing apparatus and conditions (Fig. 3e).

\section{Discussion}

We here demonstrate $\left[{ }^{11} \mathrm{C}\right] \mathrm{DASB}-\mathrm{PET}$ as a suitable and reliable method for the quantitative assessment of SERT density in selected regions of the mouse brain. We provide evidence for the application of the established approach in a longitudinal study design in a mouse model to demonstrate that the development of a behavioral phenotype associated with depression is paralleled by a dynamic regulation of SERT expression in several target brain areas. Previously it has been reported that $\left[{ }^{11} \mathrm{C}\right]$ DASB-PET can be used to determine SERT density in the rat brain, while the application to similarly accurate 


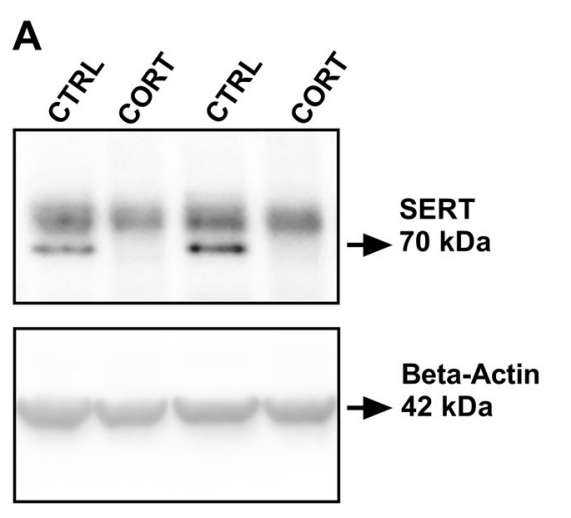

B
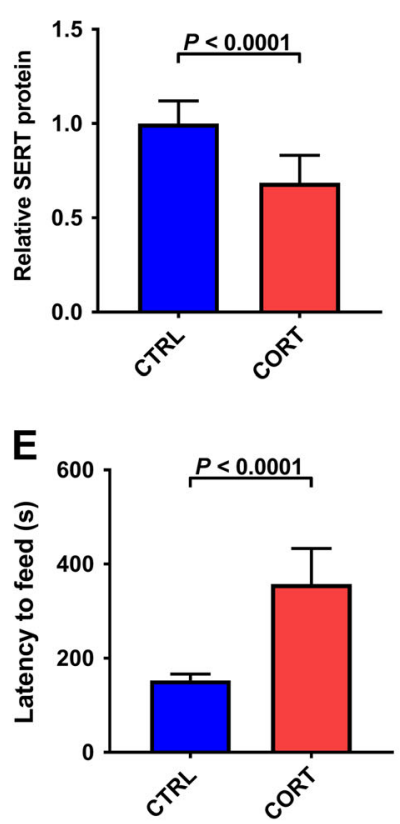

C

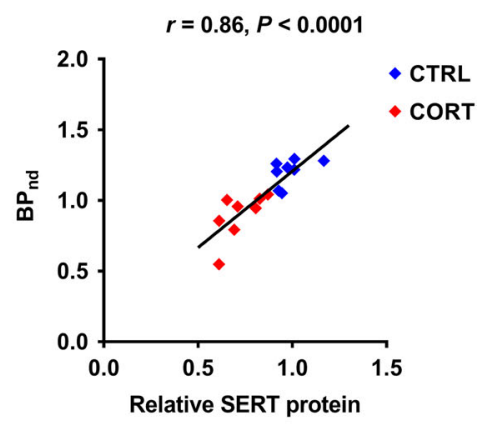

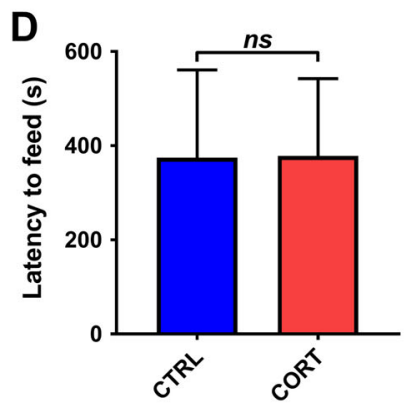

Fig. 3 Effect of CORT on hippocampal SERT levels and depression-like behavior. a Representative western blot bands and $\mathbf{b}$ quantification of relative SERT protein in hippocampal tissue of wild-type mice of the CORT and CTRL groups (Welch corrected $t$-test: $t_{15.56}=5.095, P<0.0001, n=$ 9-10). c Correlation between $\mathrm{BP}_{\mathrm{nd}}$ values measured with PET and relative SERT protein expression assessed by western blot (Pearson correlation: $r=$ $0.86, P<0.0001, n=17$ ). Depression-like behavior in the NSF $\mathbf{d}$ prior to (Welch corrected $t$-test: $\left.t_{13}=0.041, P=0.968, n=7-8\right)$ and $\mathbf{e}$ after (Welch corrected $t$-test: $\left.t_{7.58}=7.534, P<0.0001, n=7-8\right)$ chronic CORT and CTRL treatments. Data are presented as mean \pm SD. SERT serotonin transporter, CORT corticosterone treatment, CTRL control/vehicle treatment, $\mathrm{BP}_{\text {nd }}$ non-displaceable binding potential, NSF novelty-suppressed feeding test, ns nonsignificant, PET positron emission tomography

quantitative measurements in the mouse was considered difficult, most likely due to excessive levels of SERT occupancy induced by the injected mass of unlabeled $\mathrm{DASB}^{27}$. Here we met these challenges and report that by using $\left[{ }^{11} \mathrm{C}\right] \mathrm{DASB}$ with moderately improved molar activity (Table 1) when compared to the study by Walker et $\mathrm{al}^{27}$, as well as larger group sizes, we were able to measure previously reported genetic differences in SERT levels in the mouse brain $^{40}$ and to validate in vivo PET measurements by independent, ex vivo biochemical assessments.

Working within the limitations of the low spatial resolution of PET, we were able to define individual serotonergic projection brain areas with relevance for neuropsychiatric disorders related to serotonergic neurotransmission and SERT function ${ }^{40}$. However, future technological improvements in terms of image reconstruction algorithms and analyses will be needed in order to include the evaluation of additional brain regions, which may be important when determining SERT density and its dynamic changes in the mouse. In particular, the midbrain (containing the dorsal raphe nucleus, a central area of origin of serotonergic cell bodies) could not be analyzed in the present study as the dimensions of this area in the mouse is far below the spatial resolution of our PET scanner (approximately $1.4 \mathrm{~mm})^{41}$.

Although a higher degree of individual variability in SERT levels was observed mainly among male animals, no sex differences were found in the initial characterization of $\left[{ }^{11} \mathrm{C}\right]$ DASB in SERT wild-type and heterozygous mice. The regional analysis reflected the expected distribution pattern of SERT, with the highest $\left[{ }^{11} \mathrm{C}\right] \mathrm{DASB}$ binding in the thalamus and striatum, followed by the hippocampus and lower binding levels in the cortex ${ }^{35}$. We noted only minor variations in $\left[{ }^{11} \mathrm{C}\right] \mathrm{DASB}$ uptake in the cerebellum among different mouse genotypes (Supplementary Figure S1E), which suggested very low SERT expression levels in this reference tissue region ${ }^{32}$. This problem could be partly overcome in rats by using cerebellar cortex instead of whole cerebellum as a reference tissue ${ }^{32}$, which was, however, not possible in mice due to the inherent limitations of size and resolution. Therefore, a low level of SERT-specific binding of $\left[{ }^{11} \mathrm{C}\right] \mathrm{DASB}$ in the cerebellum may have introduced a bias into the analysis of our mouse 
PET data. However, the excellent correlation between PET-derived $\mathrm{BP}_{\text {nd }}$ values and SERT density measured with western blot (Supplementary Figure S5C) suggested that our methodology was suitable to quantify SERT in vivo.

The central advantage of small-animal imaging over ex vivo analyses is the possibility for longitudinal evaluations in the form of multiple measurements in the same individual allowing for the assessment of the effects of chronic interventions/environmental conditions as well as aging over time. Here we benefitted from this possibility and addressed the dynamic impact of long-term exposure to chronic CORT (mimicking chronic stress as a precipitating factor for the development of several psychiatric disorders, including depression ${ }^{42}$ ), on SERT density within an individual mouse. Notably, the repeatedmeasures design allows for the detection of small, but significant treatment effects-such as the ones seen here -which might escape detection in traditional betweengroup designs employing ex vivo analysis of brain tissue. In the context of research into the physiological effects of stress, this becomes particularly relevant: individuals can display susceptibility or resilience to different stressors $^{43,44}$, which was also observed in this study after CORT treatment (Fig. 2b-e). This may additionally mask small effects when using traditional study designs that do not include multiple measurements in a single animal.

Hence, we were able to reveal significant reductions in SERT density in the hippocampus, striatum, thalamus, and cortex after chronic CORT treatment and corroborated previous observations reporting individual behavioral responsivities upon exposure to various chronic stress paradigms ${ }^{45,46}$. The PET outcome parameter $\mathrm{BP}_{\text {nd }}$ is defined as the ratio between the available SERT density $\left(B_{\max }\right)$ and the $K_{\mathrm{D}}$ of the radiotracer. The change in $\mathrm{BP}_{\text {nd }}$ caused by CORT treatment could thus either be due to changes in either $B_{\max }$ or $K_{\mathrm{D}}$, which we were not able to distinguish in the PET analysis approach applied herein. However, as our western blot data (Fig. 3a, b) revealed a significant reduction in SERT expression after CORT treatment, it can be assumed that the observed reduction in $\mathrm{BP}_{\text {nd }}$ primarily reflected a reduction in SERT density. A small number of studies investigating the effects of chronic CORT treatment on SERT expression in ex vivo analysis of brain tissue are available. These reports provide evidence for increases ${ }^{47}$ as well as decreases of SERT brain levels ${ }^{48}$ using different CORT administration protocols. We have recently described a dramatic reduction of SERT mRNA levels in the dorsal raphe nucleus ${ }^{49}$ in a CORT paradigm comparable to the one applied in the present study. Other studies using a variety of different stress protocols including chronic mild stress, social defeat, and chronic restraint stress have also examined the impact on SERT expression with diverging results ${ }^{50-56}$ possibly due to different rodent species used and methodological differences in the different chronic stress paradigms and CORT administration protocols. However, this variation in reported results is not exclusive to animal research $^{57}$. One may speculate that the fact that interstudy variability is so high in this field may indicate a particular fragility - or indeed a particularly high degree of dynamic regulation-of the serotonergic system, and by consequence its major component SERT.

In conclusion, the present study fills an important gap, as it constitutes the first longitudinal examination of dynamic changes in SERT density in a mouse model of chronic stress. Future studies looking at SERT density at several time points during and after CORT treatment (or other chronic stress paradigms) will certainly give additional insight into the time course of SERT regulation in this context and aid to shed further light on the role of SERT in the development of stress-induced mental illnesses.

\section{Acknowledgements}

The authors would like to thank Thomas Filip, Michael Sauberer, Mathilde Löbsch, Severin Mairinger, and Johann Stanek from the AIT imaging group, as well as Marianne Ronovsky, for their help in this study. The project was supported by a stand-alone grant of the Austrian Science Fund (FWF, P28683) to D.D.P.

\section{Author details}

'Department of Neurophysiology and Neuropharmacology, Center for Physiology and Pharmacology, Medical University of Vienna,

Schwarzspanierstraße 17, 1090 Vienna, Austria. ${ }^{2}$ Biomedical Systems, AIT Austrian Institute of Technology GmbH, 2444 Seibersdorf, Austria. . ${ }^{3}$ Department of Clinical Pharmacology, Medical University of Vienna, Währinger Gürtel 18-20, 1090 Vienna, Austria. ${ }^{4}$ Department of Biomedical Imaging und Image-guided Therapy, Division of Nuclear Medicine, Medical University of Vienna, Währinger Gürtel 18-20, 1090 Vienna, Austria

\section{Conflict of interest}

The authors declare that they have no conflict of interest.

\section{Publisher's note}

Springer Nature remains neutral with regard to jurisdictional claims in published maps and institutional affiliations.

Supplementary information accompanies this paper at (https://doi.org/ 10.1038/s41398-019-0416-7).

Received: 23 August 2018 Revised: 8 December 2018 Accepted: 17 January 2019

Published online: 11 February 2019

\footnotetext{
References

1. Fakhoury, M. Revisiting the serotonin hypothesis: implications for major depressive disorders. Mol. Neurobiol. 53, 2778-2786 (2016).

2. López-Figueroa, A. L. et al. Serotonin 5-HT1A, 5-HT1B, and 5-HT2A receptor mRNA expression in subjects with major depression, bipolar disorder, and schizophrenia. Biol. Psychiatry 55, 225-233 (2004).

3. Davidson, R. J. et al. Neural and behavioral substrates of mood and mood regulation. Biol. Psychiatry 52, 478-502 (2002).

4. McGinty, D. \& Szymusiak, R. The sleep-wake switch: a neuronal alarm clock. Nat. Med. 6, 510-511 (2000).
} 
5. Monti, J. M. Serotonin control of sleep-wake behavior. Sleep Med. Rev. 15, 269-281 (2011)

6. Ferrari, P. F., Palanza, P., Parmigiani, S., de Almeida, R. M. M. \& Miczek, K. A. Serotonin and aggressive behavior in rodents and nonhuman primates: predispositions and plasticity. Eur. J. Pharmacol. 526, 259-273 (2005).

7. Monteleone, P., Brambilla, F., Bortolotti, F. \& Maj, M. Serotonergic dysfunction across the eating disorders: relationship to eating behaviour, purging behaviour, nutritional status and general psychopathology. Psychol. Med. 30, 1099-1110 (2000).

8. Leibowitz, S. F. The role of serotonin in eating disorders. Drugs 39(Suppl 3), 33-48 (1990).

9. Luo, M., Zhou, J. \& Liu, Z. Reward processing by the dorsal raphe nucleus: 5-HT and beyond. Learn. Mem. 22, 452-460 (2015).

10. Liu, Z. et al. Dorsal raphe neurons signal reward through 5-HT and glutamate. Neuron 81, 1360-1374 (2014).

11. Fischer, A. G. \& Ullsperger, M. An update on the role of serotonin and its interplay with dopamine for reward. Front. Hum. Neurosci. 11, 484 (2017).

12. Heinz, A., Mann, K., Weinberger, D. R. \& Goldman, D. Serotonergic dysfunction, negative mood states, and response to alcohol. Alcohol. Clin. Exp. Res. 25, 487-495 (2001)

13. Heinz, A. et al. Reduced central serotonin transporters in alcoholism. Am. J. Psychiatry 155, 1544-1549 (1998).

14. Homberg, J. R., Schubert, D., Asan, E. \& Aron, E. N. Sensory processing sensitivity and serotonin gene variance: insights into mechanisms shaping environmental sensitivity. Neurosci. Biobehav. Rev. 71, 472-483 (2016).

15. Brown, G. W. \& Harris, T. O. Depression and the serotonin transporter 5-HTTLPR polymorphism: a review and a hypothesis concerning gene-environment interaction. J. Affect Disord. 111, 1-12 (2008).

16. Zhang, $\mathrm{K}$. et al. The combined effects of the 5-HTTLPR and 5-HTR1A genes modulates the relationship between negative life events and major depressive disorder in a Chinese population. J. Affect Disord. 114, 224-231 (2009).

17. Klengel, T. \& Binder, E. B. Gene-environment interactions in major depressive disorder. Can. J. Psychiatry 58, 76-83 (2013).

18. Caspi, A., Hariri, A. R., Holmes, A., Uher, R. \& Moffitt, T. E. Genetic sensitivity to the environment: the case of the serotonin transporter gene and its implications for studying complex diseases and traits. Am. J. Psychiatry 167, 509-527 (2010).

19. Schloss, P. \& Williams, D. C. The serotonin transporter: a primary target for antidepressant drugs. J. Psychopharmacol. 12, 115-121 (1998).

20. Hariri, A. R. \& Holmes, A. Genetics of emotional regulation: the role of the serotonin transporter in neural function. Trends Cogn. Sci. 10, 182-191 (2006).

21. Insel, $T$. et al. Research domain criteria (RDoC): toward a new classification framework for research on mental disorders. Am. J. Psychiatry 167, 748-751 (2010).

22. Berton, O., Hahn, C.-G. \& Thase, M. E. Are we getting closer to valid translational models for major depression? Science 338, 75-79 (2012)

23. Houle, S., Ginovart, N., Hussey, D., Meyer, J. H. \& Wilson, A. A. Imaging the serotonin transporter with positron emission tomography: initial human studies with [11C]DAPP and [11C]DASB. Eur. J. Nucl. Med. 27, 1719-1722 (2000).

24. Frankle, W. G. et al. Estimation of serotonin transporter parameters with $11 \mathrm{C}$ DASB in healthy humans: reproducibility and comparison of methods. J. Nucl. Med. 47, 815-826 (2006).

25. Kim, J. S., Ichise, M., Sangare, J. \& Innis, R. B. PET imaging of serotonin transporters with [11C]DASB: test-retest reproducibility using a multilinear reference tissue parametric imaging method. J. Nucl. Med. 47, 208-214 (2006).

26. Huang, $Y$. et al. Comparative evaluation in nonhuman primates of five PET radiotracers for imaging the serotonin transporters: $\left[1^{1} \mathrm{C}\right] \mathrm{MCN} 5652,\left[1^{1} \mathrm{C}\right]$ ADAM, $\left[1^{1}\right.$ C]DASB, $\left[1^{1}\right.$ C]DAPA, and $\left[1^{1}\right.$ C AFM. J. Cereb. Blood Flow. Metab. 22, 1377-1398 (2002)

27. Walker, M., Ehrlichmann, W., Stahlschmidt, A., Pichler, B. J. \& Fischer, K. In vivo evaluation of 11C-DASB for quantitative SERT imaging in rats and mice. J. NuCl. Med. 57, 115-121 (2016).

28. Wilson, A. A. et al. Novel radiotracers for imaging the serotonin transporter by positron emission tomography: synthesis, radiosynthesis, and in vitro and ex vivo evaluation of (11)C-labeled 2-(phenylthio)araalkylamines. J. Med. Chem. 43, 3103-3110 (2000).

29. Haeusler, D. et al. Simple and rapid preparation of [11C]DASB with high quality and reliability for routine applications. Appl. Radiat. Isot. 67, 1654-1660 (2009).

30. Gourley, S. L. \& Taylor J. R. Recapitulation and reversal of a persistent depression-like syndrome in rodents. Curr. Protoc. Neurosci. Chapter 9, Unit 9.32 (2009).
31. Britton, D. R. \& Britton, K. T. A sensitive open field measure of anxiolytic drug activity. Pharmacol. Biochem. Behav. 15, 577-582 (1981).

32. Mineur, Y. S., Picciotto, M. R. \& Sanacora, G. Antidepressant-like effects of ceftriaxone in male C57BL/6J mice. Biol. Psychiatry 61, 250-252 (2007).

33. Fischer, $\mathrm{K}$. et al. Noninvasive nuclear imaging enables the in vivo quantification of striatal dopamine receptor expression and raclopride affinity in mice. J. NuCl. Med. 52, 1133-1141 (2011).

34. Griesauer, I. et al. Circadian abnormalities in a mouse model of high trait anxiety and depression. Ann. Med. 46, 148-154 (2014).

35. Bengel, D. et al. Cellular localization and expression of the serotonin transporter in mouse brain. Brain Res. 778, 338-345 (1997).

36. Deltheil, T. et al. Behavioral and serotonergic consequences of decreasing or increasing hippocampus brain-derived neurotrophic factor protein levels in mice. Neuropharmacology 55, 1006-1014 (2008).

37. Homberg, J. R., Molteni, R., Calabrese, F. \& Riva, M. A. The serotonin-BDNF duo: developmental implications for the vulnerability to psychopathology. Neurosci. Biobehav. Rev. 43, 35-47 (2014).

38. Mahar, I., Bambico, F. R., Mechawar, N. \& Nobrega, J. N. Stress, serotonin, and hippocampal neurogenesis in relation to depression and antidepressant effects. Neurosci. Biobehav. Rev. 38, 173-192 (2014).

39. David, D. J. et al. Neurogenesis-dependent and -independent effects of fluoxetine in an animal model of anxiety/depression. Neuron 62, 479-493 (2009).

40. Montgomery, T. R. et al. Axonal targeting of the serotonin transporter in cultured rat dorsal raphe neurons is specified by SEC24C-dependent export from the endoplasmic reticulum. J. Neurosci. 34, 6344-6351 (2014).

41. Tai, Y.-C. et al. Performance evaluation of the microPET focus: a thirdgeneration microPET scanner dedicated to animal imaging. J. Nucl. Med. 46, 455-463 (2005).

42. Kendler, K. S., Karkowski, L. M. \& Prescott, C. A. Causal relationship between stressful life events and the onset of major depression. Am. J. Psychiatry 156, 837-841 (1999).

43. Hodes, G. E. et al. Individual differences in the peripheral immune system promote resilience versus susceptibility to social stress. Proc. Natl Acad. Sci. USA 111, 16136-16141 (2014).

44. Kim, J.-G., Jung, H.-S., Kim, K.-J., Min, S.-S. \& Yoon, B.-J. Basal blood corticosterone level is correlated with susceptibility to chronic restraint stress in mice. Neurosci. Lett. 555, 137-142 (2013).

45. Franklin, T. B., Saab, B. J. \& Mansuy, I. M. Neural mechanisms of stress resilience and vulnerability. Neuron 75, 747-761 (2012).

46. Russo, S. J., Murrough, J. W., Han, M.-H., Charney, D. S. \& Nestler, E. J. Neurobiology of resilience. Nat. Neurosci. 15, 1475-1484 (2012).

47. Zhang, J. et al. Chronic social defeat up-regulates expression of the serotonin transporter in rat dorsal raphe nucleus and projection regions in a glucocorticoid-dependent manner. J. Neurochem. 123, 1054-1068 (2012).

48. Tang, M. et al. Subregion-specific decreases in hippocampal serotonin transporter protein expression and function associated with endophenotypes of depression. Hippocampus 24, 493-501 (2014).

49. Reisinger, S. N. et al. Flotillin-1 interacts with the serotonin transporter and modulates chronic corticosterone response. Genes Brain Behav. e12482 (2018).

50. Berton, O., Durand, M., Aguerre, S., Mormède, P. \& Chaouloff, F. Behavioral, neuroendocrine and serotonergic consequences of single social defeat and repeated fluoxetine pretreatment in the Lewis rat strain. Neuroscience $\mathbf{9 2}$, 327-341 (1999).

51. McKittrick, C. R. et al. Chronic social stress reduces dendritic arbors in CA3 of hippocampus and decreases binding to serotonin transporter sites. Synapse 36, 85-94 (2000)

52. Morozova, A. Y., Zubkov, E. A., Koshkin, F. A., Storozheva, Z. I. \& Chekhonin, V. P. Expression of genes encoding serotonin receptors and SERT in various brain structures of stressed rats after chronic exposure to ultrasound. Bull. Exp. Biol. Med. 156, 317-319 (2014)

53. Filipenko, M. L., Beilina, A. G., Alekseyenko, O. V., Dolgov, V. V. \& Kudryavtseva, N. $\mathrm{N}$. Repeated experience of social defeats increases serotonin transporter and monoamine oxidase A mRNA levels in raphe nuclei of male mice. Neurosci. Lett. 321, 25-28 (2002).

54. Gardner, K. L., Hale, M. W., Lightman, S. L., Plotsky, P. M. \& Lowry, C. A. Adverse early life experience and social stress during adulthood interact to increase serotonin transporter mRNA expression. Brain Res. 1305, 47-63 (2009).

55. Abumaria, N. et al. Identification of genes regulated by chronic social stress in the rat dorsal raphe nucleus. Cell. Mol. Neurobiol. 26, 145-162 (2006). 
56. Vollmayr, B., Keck, S., Henn, F. A. \& Schloss, P. Acute stress decreases serotonin transporter mRNA in the raphe pontis but not in other raphe nuclei of the rat. Neurosci. Lett. 290, 109-112 (2000).
57. Spies, M., Knudsen, G. M., Lanzenberger, R. \& Kasper, S. The serotonin transporter in psychiatric disorders: insights from PET imaging. Lancet Psychiatry 2, 743-755 (2015). 